\title{
Hot carrier solar cell absorbers: materials, mechanisms and nanostructures
}

Gavin Conibeer, Santosh Shrestha, Shujuan Huang, Robert Patterson, Hongze Xia, et al.

Gavin Conibeer, Santosh Shrestha, Shujuan Huang, Robert Patterson, Hongze Xia, Yu Feng, Pengfei Zhang, Neeti Gupta, Murad Tayebjee, Suntrana Smyth, Yuanxun Liao, Zhilong Zhang, Simon Chung, Shu Lin, Pei Wang, Xi Dai, "Hot carrier solar cell absorbers: materials, mechanisms and nanostructures," Proc. SPIE 9178, Next Generation Technologies for Solar Energy Conversion V, 917802 (17 October 2014); doi: 10.1117/12.2067926

SPIE Event: SPIE Solar Energy + Technology, 2014, San Diego, California, United States 


\title{
Hot Carrier solar cell absorbers: materials, mechanisms and nanostructures
} Gavin Conibeer ${ }^{1}$, Santosh Shrestha ${ }^{1}$, Shujuan Huang ${ }^{1}$, Robert Patterson ${ }^{1}$, Hongze Xia ${ }^{1}$, Yu Feng ${ }^{1}$, Pengfei Zhang ${ }^{1}$, Neeti Gupta ${ }^{1}$, Murad Tayebjee ${ }^{1}$, Suntrana Smyth ${ }^{1}$, Yuanxun Liao ${ }^{1}$, Zhilong Zhang ${ }^{1}$, Simon Chung ${ }^{1}$, Shu Lin ${ }^{1}$, Pei Wang ${ }^{1}$, Xi Dai ${ }^{1}$

${ }^{1}$ Australian Centre for Advanced Photovoltaics, School of Photovoltaic and Renewable Energy Engineering, The University of New South Wales, NSW, Australia

\section{Corresponding author: Gavin Conibeer}

Phone number: +61293857858

Fax number: +61293855456

E-mail address: g.conibeer@unsw.edu.au

Postal address: TETB (building H6), University of New South Wales, Sydney, NSW 2052, Australia

\begin{abstract}
The hot carrier cell aims to extract the electrical energy from photo-generated carriers before they thermalize to the band edges. Hence it can potentially achieve a high current and a high voltage and hence very high efficiencies up to $65 \%$ under 1 sun and $86 \%$ under maximum concentration. To slow the rate of carrier thermalisation is very challenging, but modification of the phonon energies and the use of nanostructures are both promising ways to achieve some of the required slowing of carrier cooling. A number of materials and structures are being investigated with these properties and test structures are being fabricated. Initial measurements indicate slowed carrier cooling in III-Vs with large phonon band gaps and in multiple quantum wells. It is expected that soon proof of concept of hot carrier devices will pave the way for their development to fully functioning high efficiency solar cells.
\end{abstract}

Keywords:

phonons; carrier cooling; hot carriers; time resolved photoluminescence; Klemens decay; multiple quantum wells

\section{INTRODUCTION}

The hot carrier solar cell is a solar energy converter that utilizes the excess thermal energy of photo-excited carriers to generate DC electric power. Unlike conventional solar cells, the hot carrier cell maintains the hot carrier population by inhibiting ultrafast cooling processes. The excess carrier energies above the respective band edges thus contribute to a higher conversion efficiency than that of a conventional cell, which is restricted by the detailed-balance (ShockleyQueisser) limit for a single junction cell to $33 \%$. The hot carrier cell has a limiting efficiency of $66 \%$ for unconcentrated sunlight rising to $85 \%$ at maximum concentration (46,200 suns), assuming ideal operation under detailed balance. ${ }^{1,2,3}$

An ideal Hot Carrier cell would absorb a wide range of photon energies and extract a large fraction of the energy to give very high efficiencies by extracting 'hot' carriers before they thermalise to the band edges. Hence an important property of a hot carrier cell is to slow the rate of carrier cooling to allow hot carriers to be collected whilst they are still at elevated energies ("hot"), and thus allowing higher voltages to be achieved from the cell and hence higher efficiency. A Hot Carrier cell must also only allow extraction of carriers from the device through contacts that accept only a very narrow range of energies (energy selective contacts or ESCs). This is necessary in order to prevent cold carriers in the contact from cooling the hot carriers, i.e. the increase in entropy on carrier extraction is minimized. ${ }^{4,5}$

Fig. 1 is a schematic band diagram of a Hot Carrier cell illustrating these requirements. ${ }^{6}$ 


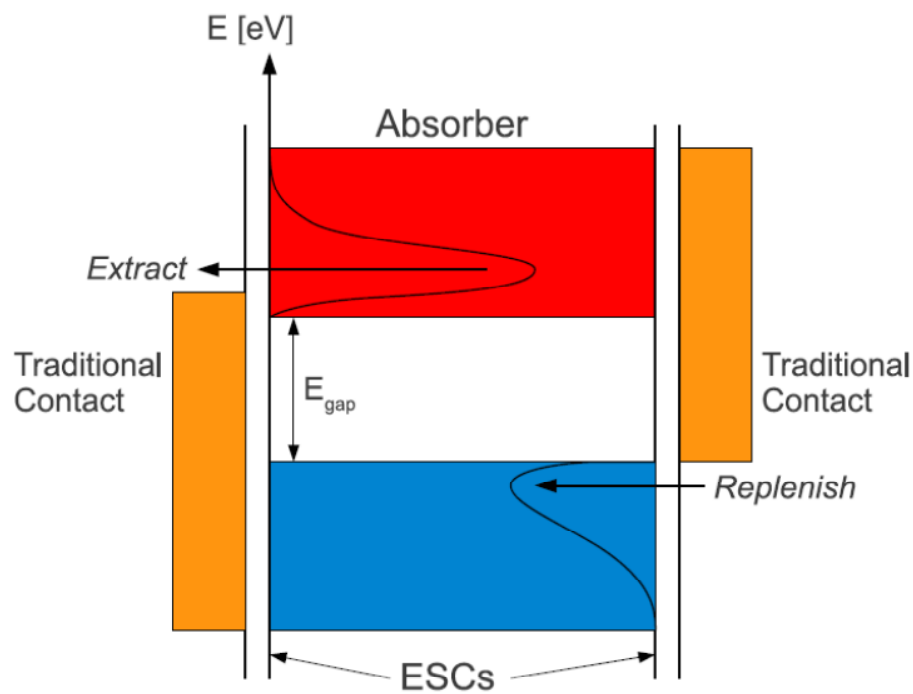

Figure 1: Band diagram of the Hot Carrier cell: carriers remain at elevated temperature in the absorber and are extracted through narrow band energy selective contacts at high voltage. ${ }^{6}$ The device has four stringent requirements: a) To absorb a wide range of photon energies; b) to slow the rate of photogenerated carrier cooling in the absorber; c) To extract these 'hot carriers' over a narrow range of energies, such that excess carrier energy is not lost to the cold contacts; d) to allow efficient renormalisation of carrier energy in the absorber via carrier-carrier scattering.

\section{SLOWING OF CARRIER COOLING}

The energy dissipation of photo-generated carriers is a multi-step process. The inelastic scattering of carriers takes place in the first few 10 s of femtoseconds after their generation. This process normalizes momentum and leads to a distribution of electron energies which can be described by a Boltzman distribution and a single high temperature, i.e. a thermal population, and a separate (generally lower temperature) thermal population of holes. ${ }^{7}$ Then on a longer timescale (typically several picoseconds) carriers scatter inelastically with phonons, predominantly emitting optical phonons in a series of discrete hops in each of which energy and momentum are conserved in the combination of electron and emitted phonon. For polar semiconductors the major scattering process is with longitudinal optical phonon modes. These optical phonons emitted by the excited carriers then interact with other phonons due to the anharmonic nature of the crystal potential. Through various routes these over-populated optical phonons decay into low-energy acoustic phonons. The final step is the transport of these acoustic phonons, macroscopically manifest as the heat dissipation to the environment. The carrier cooling process can be slowed by blocking any of these three processes. Other processes, such as direct emission of acoustic phonons and diffusion of optical phonons, are not significant for polar semiconductors.

The cooling of carriers by emission of optical phonons leads to the build-up of a non-equilibrium 'hot' population of optical phonons which, if it remains hot, will drive a reverse reaction to re-heat the carrier population, thus slowing further carrier cooling. Therefore the critical factor is the mechanism by which these optical phonons decay into acoustic phonons, or heat in the lattice. The principal mechanism by which this can occur is the Klemens mechanism, in which the optical phonon decays into two acoustic phonons of half its energy and of equal and opposite momenta. ${ }^{8}$ The build up of emitted optical phonons is strongly peaked at zone centre both for compound semiconductor due to the Fröhlich interaction (strong quadratic dependence on momentum) and for elemental semiconductors due to the deformation potential interaction (less strong linear dependence on momentum). The strong coupling of the Fröhlich interaction also means that high energy optical phonons are also constrained to near zone centre even if parabolicty of the bands is no longer valid as is the case for high energy carriers well above the band minima. ${ }^{9}$ This zone centre optical phonon population determines that the dominant optical phonon decay mechanism is this pure Klemens decay.

\subsection{Phonon decay mechanisms}

An optical phonon will decay into multiple lower energy phonons. The processes require the conservation of energy and momentum. However, additionally, the principal decay path must be into two LA phonons only, which are of equal energy and opposite momenta, via an anharmonicity in the lattice, this was suggested by Klemens ${ }^{8}$ and has been shown to apply to a wide range of materials. 


\subsubsection{Wide phononic gaps in III-Vs and analogues}

For some compounds in which there is a large difference in masses of the constituent elements, there exists a large gap in the phonon dispersion between high-lying optical phonon energies and low-lying acoustic phonon energies. If large enough this "phononic band gap" can prevent Klemens decay of optical phonons, because no allowed states at half the LO phonon energy exist. Indium nitride $(\mathrm{InN})$ is an example of such a material with a very large phonon gap.

This prevention of the Klemens mechanism forces optical phonon decay via the next most likely, Ridley mechanism, of emission of one TO and one low energy LA phonon. Such a mechanism only has appreciable energy loss (although still much less than Klemens' decay) if there is a wide range of optical phonon energies at zone centre. This is only the case for lower symmetry structures such as hexagonal. For a high symmetry cubic structure, LO and TO modes are close to degenerate at zone centre with a very flat dispersion and the Ridley mechanism is severely restricted or forbidden. Unfortunately cubic InN is very difficult to fabricate precisely because of the large difference in masses that give it its interesting phononic dispersion. In addition to the small dispersion in cubic structures dispersionless structures can be achieved by engineering nanostructures.

Slowed cooling has been observed in some III-V compounds in which there is a large difference in atomic mass. This has been shown in slowed carrier cooling for $\mathrm{InN}^{10}$ as plotted in Fig. 2, and for slowed carrier cooling in InP compared to the small mass ratio GaAs, ${ }^{11}$ as discussed below in section 4. Analogues of $\mathrm{InN}$ with abundant elements, but also with narrow $\mathrm{E}_{\mathrm{g}}$ are discussed below in Section 3.1.

\subsection{Nanostructures for the absorber}

Nanostructures are good options to fulfill the requirements of the hot carrier absorber. Two types of nanostructures have been suggested: nanowell structure and nanodot structures. The nanowell structure has very thin layers of narrow band gap materials stacked in layers separated by potential barriers, creating oscillating potentials for the electrons. The electronic dispersion relations become flat for energies below the barrier height. This can potentially reduce the rate of electron-phonon polar interactions.

\subsubsection{Slowed carrier cooling in MQWs}

Low dimensional multiple quantum well (MQW) systems have also been shown to have lower carrier cooling rates.

Comparison of bulk and MQW materials has shown significantly slower carrier cooling in the latter. Figure shows data for bulk GaAs as compared to MQW GaAs/AlGaAs materials as measured using time resolved transient absorption by Rosenwaks et. al. ${ }^{12}$ recalculated to show effective carrier temperature as a function of carrier lifetime by Guillemoles. ${ }^{13}$ This clearly shows that the carriers stay hotter for significantly longer times in the MQW samples, particularly at the higher injection levels by $1 \frac{1}{2}$ orders of magnitude. This is due to an enhanced 'phonon bottleneck' in the MQWs allowing the threshold intensity at which a certain ratio of LO phonon re-absorption to emission is reached which allows maintenance of a hot carrier population, to be reached at a much lower illumination level. More recent work on strain balanced InGaAs/GaAsP MQWs by Hirst ${ }^{14}$ has also shown carrier temperatures significantly above ambient, as measured by photoluminescence. Increase in In content to make the wells deeper and to reduce the degree of confinement is seen to increase the effective carrier temperatures. ${ }^{15}$ And extraction of a small portion of this nonequilibrium hot carrier population as a very small external current at low temperatures is demonstrated for a single InGaAs QW by Hirst. ${ }^{16}$

The mechanisms for the reduced carrier cooling rate in these MQW systems are not yet clear. However there are at least three possible effects that are likely to contribute, more than one of which could well occur in parallel. The first of these effects is that in bulk material photogenerated hot carriers are free to diffuse deeper into the material and hence to reduce the hot carrier concentration at a given depth. This will also decrease the density of LO phonons emitted by hot carriers as they cool and make a phonon bottleneck more difficult to achieve at a given illumination intensity. Whereas in a MQW there are physical barriers to the diffusion of hot carriers generated in a well and hence a much greater local concentration of carriers and therefore also of emitted optical phonons. Thus the phonon bottleneck condition is achieved at lower intensity.

The second effect is that for the materials systems which show this slowed cooling, there is very little or no overlap between the optical phonon energies of the well and barrier materials. For instance the optical phonon energy ranges for 
the GaAs wells and AlGaAs barriers used in ${ }^{12}$ at $210-285 \mathrm{~cm}^{-1}$ and $280-350 \mathrm{~cm}^{-1}$, respectively, exhibit very little overlap in energy, with zero overlap for the zone centre LO phonon energies of 285 and $350 \mathrm{~cm}^{-1} .{ }^{17}$ Consequently the predominantly zone centre LO phonons emitted by carriers cooling in the wells will be reflected from the interfaces and will remain confined in the wells, thus enhancing the phonon bottleneck at a given illumination intensity.

Thirdly, if there is a coherent spacing between the nano-wells (as there is for these MQW or superlattice systems) a coherent Bragg reflection of phonon modes can be established which blocks certain phonon energies perpendicular to the wells, opening up one dimensional phononic band gaps (analogous to photonic band gaps in modulated refractive index structures. For specific ranges of nano-well and barrier thickness these forbidden energies can be at just those energies required for phonon decay. This coherent Bragg reflection should have an even stronger effect than the incoherent scattering of the second mechanism above at preventing emission of phonons and phonon decay in the direction perpendicular to the nano-wells.

It is likely that all three of these effects will reduce carrier cooling rates. None depend on electronic quantum confinement and hence should be exhibited in wells that are not thin enough to be quantized but are still quite thin (perhaps termed 'nano-wells'). In fact it may well be that the effects are enhanced in such nano-wells as compared to full QWs due to the former's greater density of states and in particular their greater ratio of density of electronic to phonon states which will enhance the phonon bottleneck for emitted phonons. The fact that the deeper and hence less confined wells in ${ }^{14}$ show higher carrier temperatures is tentative evidence to support the hypothesis that nano-wells without quantum confinement are all that are required. Whilst several other effects might well be present in these MQW systems, further work on variation of nano-well and barrier width and comparison between material systems, will distinguish which of these reduced carrier diffusion, phonon confinement or phonon folding mechanisms might be dominant.

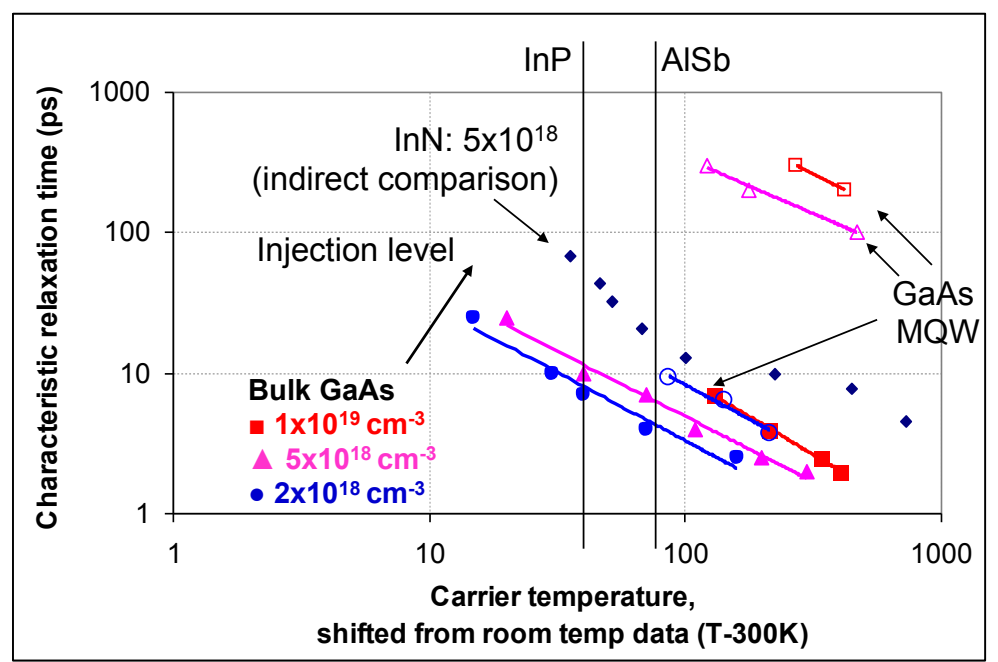

Figure 2: Effective carrier temperature as a function of carrier lifetime for bulk GaAs as compared to GaAs/AlGaAs MQWs: time resolved transient absorption data for different injection levels, from Rosenwaks, ${ }^{12}$ recalculated by Guillemoles. ${ }^{13}$ Also shown are temperatures from transient absorption data for InN from Chen ${ }^{10}$ and indicative carrier temperatures based on long phonon lifetimes for AlSb and InP from Barman. ${ }^{26}$

\subsection{Hot Carrier cell absorber requisite properties}

The above discussion allows us to estimate the major properties required for a good hot carrier absorber material. These are listed below in approximate order of priority, although their relative importance may well change in light of future research:

1. Large phononic band gap $\left(E_{O}(\mathrm{~min})-E_{L A}\right)$ - to suppress Klemens' decay, requires large mass difference between elements.

2. Narrow optical phonon energy dispersion $\left(E_{L O}-E_{O}(\mathrm{~min})\right)$ - to minimise the loss by Ridley decay, requires a high symmetry.

3. Small $E_{g}<1 \mathrm{eV}$ - to allow a broad range of strong photon absorption. 
4. A small LO optical phonon energy $\left(E_{L O}\right)$ - to reduce the amount of energy lost per LO phonon emission but not so small as to increase the occupancy of LO modes unduly. $E_{L O} \cdot \exp \left(-E_{L O} / k T\right)$

5. A small maximum acoustic phonon energy $\left(E_{L A}\right)$. This maximises $\left(E_{O}(\mathrm{~min})-E_{L A}\right)$ and is important if $E_{L O}$ is also small.

6. Good renormalisation rates in the material, i.e. good $e-e$ and $h-h$ scattering. This condition is met in most semiconductors quite easily, with e-e scattering rates of less than 100fs. But it may be compromised in nanostructures.

7. Good carrier transport in order to allow transport of hot carriers to the contacts.

8. Ability to make good quality, ordered, low defect material.

9. Earth abundant and readily processable materials.

10. No, or low, toxicity of elements, compounds and processes.

\section{Hot Carrier Absorber: Choice of Materials}

InN has most of these properties, except 4, $8 \& 9$, and is therefore a good model material for a hot carrier cell absorber. However the abundance of In is very low, so it is difficult to see $\mathrm{InN}$ as a long term material suitable for large scale implementation. Hence analogues of InN, which retain its interesting property of a large phonon band gap, are also being investigated.

\subsection{Analogues of $\operatorname{In} N$}

As InN is a model material, but has the problems of abundance and bad material quality, another approach is to use analogues of InN to attempt to emulate its near ideal properties. These analogues can be II-IV-nitride compounds, other nitrides or possibly oxides, large mass anion III-Vs, group IV compounds/alloys or nanostructures.

IIA IIIA IB IIB IIIB IVB $\quad$ VB $\quad$ VIB

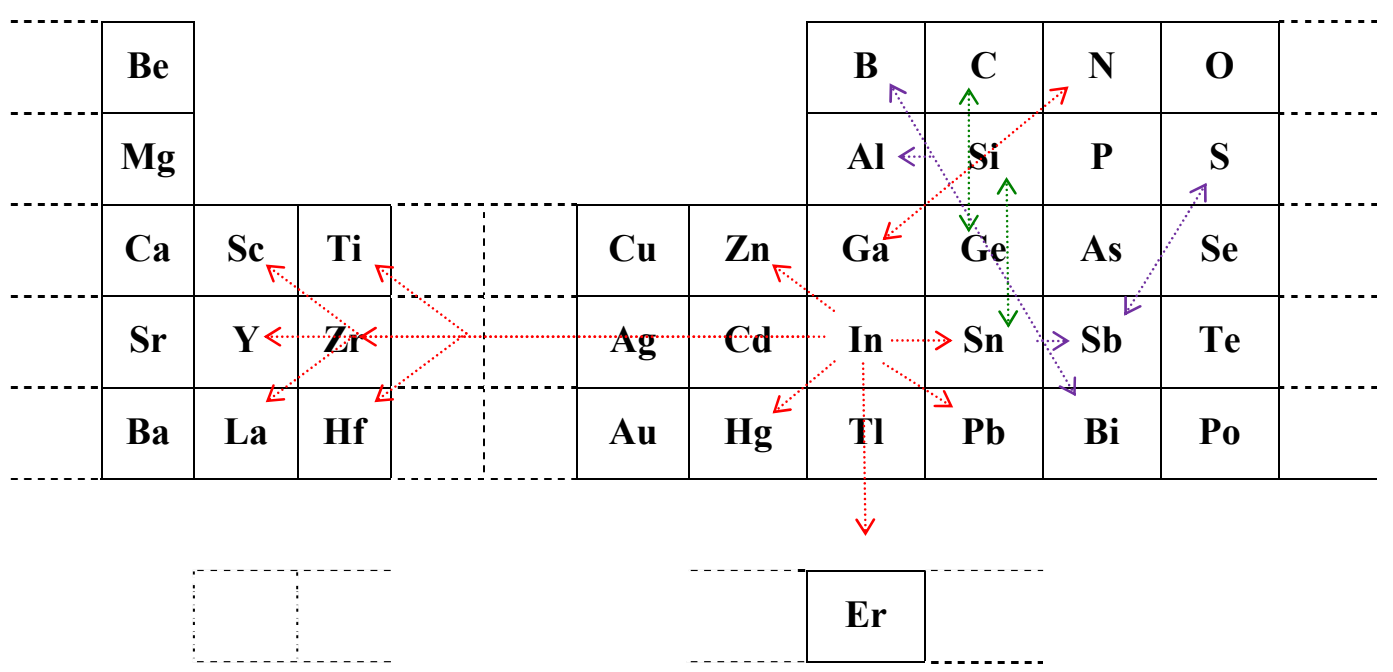

Figure 3: Use of the periodic table to analyse possible analogue compounds of InN based on atomic mass combination and electronegativity.

\subsubsection{II-IV-Vs: ZnSnN; ZnPbN; HgSnN; HgPbN}

With reference to Figure 3, it can be seen that replacement of In on the III sub-lattice with II-IV compounds is analogous and is now quite widely being investigated in the $\mathrm{Cu}_{2} \mathrm{ZnSnS}_{4}$ analogue to $\mathrm{CuInS}_{2}{ }^{18}$

$\mathrm{ZnGeN}$ can be fabricated ${ }^{19,20}$ and is most directly analogous with Si and GaAs. However, its electronic band gap is large at $1.9 \mathrm{eV}$. It also has a small calculated phononic band gap. ${ }^{21} \mathrm{ZnSnN}$ has a smaller electronic gap (1 eV) and larger calculated phononic gap. ${ }^{20,21}$ It is however difficult to fabricate, and also its phononic gap is not as large as the acoustic phonon energy making it difficult to block Klemens decay completely. $\mathrm{HgSnN}$ or $\mathrm{HgPbN}$ should both have smaller $\mathrm{E}_{\mathrm{g}}$ 
and larger phononic gaps. These materials have not yet been fabricated and have the problem that Hg has about half the abundance of In and is toxic. ${ }^{22}$

\subsubsection{Large mass anion:}

$\mathrm{Bi}$ and $\mathrm{Sb}$ are heavy elements and hence their compounds with $\mathrm{B}$ and $\mathrm{Al}$ have large predicted phononic gaps. ${ }^{23} \mathrm{BiB}$ has the largest phononic gap but $\mathrm{AlBi}, \mathrm{Bi}_{2} \mathrm{~S}_{5}, \mathrm{Bi}_{2} \mathrm{O}_{3}$ (bismuthine) are also attractive. AlSb has a calculated and measured phononic gap the same size as its acoustic phonon energy. ${ }^{24}$ It also has a very flat optical phonon dispersion that should suppress Ridely as well as there being almost no chance of Klemens decay. ${ }^{25}$ ASb has a very high calculated phonon lifetime probably because of its large phononic gap, similar to $\mathrm{InP},{ }^{26}$ which give the predicted large carrier temperatures shown in Fig. 2. The electronic band gap of AlSb is $1.5 \mathrm{eV}$ which, although indirect, makes it marginally suitable as an absorber. These phonon properties, and the fact that it is of reasonably widespread use and can be made of reasonable quality, make AlSb reasonably attractive as a hot carrier absorber material. BSb has a larger elemental mass difference giving it a very large phonon band gap more than twice the acoustic phonon energy, as calculated in 3D using an abinitio DFT approach, ${ }^{27}$ sufficient to block Klemens decay completely if the material quality is high enough. It also can be fabricated by co-evaporation and has a measured $\mathrm{E}_{\mathrm{g}}$ of $0.59 \mathrm{eV} .^{28}$ Its band gap is indirect and hence not ideal, but the absorption coefficient remains high even out to $1700 \mathrm{~nm}$. Bi is a rare element with an earth abundance about twice that of In, with Sb about five times In's abundance. ${ }^{22}$ Nonetheless their large phonon gaps make particularly BSb and AlSb interesting to pursue further for proof of concept. A quantum well or quantum dot nanostructure of AlSb/BSb would be particularly interesting as it could combine phononic band gap and MQW slowed carrier cooling effects. Test structures in related GaInAsSb/AlGaAsSb MQW systems have indeed shown potential for reduced cooling due to reflection of phonons at interfaces. ${ }^{29}$

\subsubsection{Transition metal nitrides:}

The Group IIIA nitrides LaN, YN and ScN have large elemental mass differences and would have been thought to have large phononic gaps, but as they form in a $\mathrm{NaCl}$ structure rather than the zinc-blende structure of the Group IIIB nitrides, they actually have zero phonon band gap. ${ }^{30,31}$ However the IVA nitrides of Hafnium and Zirconium have large predicted and measured phononic gaps, ${ }^{30}$ both bigger than their acoustic phonon energies. The phonon gap for TaN is reasonable but not as large as its acoustic phonon energy. Hf and Ta are about the same abundance as Ge and As, with Zr 100x more abundant and all are widely used metals. ${ }^{22} \mathrm{Hf}_{3} \mathrm{~N}_{4}$ also shows a large phonon band gap (although still too small to block Klemens decay completely) ${ }^{32}$ but also a narrow optical phonon dispersion - important to block secondary Ridley decay. These nitrides can also readily be fabricated and have many existing applications, although the lack of stability of HfN in moist air would need to be managed in a real application, probably by encapsulation. Interestingly both $\mathrm{Hf}_{1} \mathrm{~N}_{1}$ and $\mathrm{Zr}_{1} \mathrm{~N}_{1}$ have zero electronic band gap, leading to the surprising conclusion that these semi-metals may be good absorbers for a hot carrier cell.

The Lanthanides can also form III-nitrides. ErN and other rare earth nitrides can be grown by MBE. The phononic band gaps of the Er compounds are predicted to be large, because of the heavy Er cation, but its discrete energy levels make it not useful as an absorber, although the combination of properties in a nanostructure could be advantageous.

\subsubsection{Group IV alloy/compounds:}

All of the combinations $\mathrm{Si} / \mathrm{Sn}, \mathrm{Ge} / \mathrm{C}, \mathrm{Sn} / \mathrm{C}$ or $\mathrm{Sn} / \mathrm{Si}$ look attractive with large phonon gaps predicted in an approach using perturbation DFT, ${ }^{25}$ with that for SnSi particularly large and with a very flat dispersion, which should enable it to suppress most phonon decay mechanisms. The predominantly covalent bonding, due to all the elements being group IV, results in very little splitting of optical modes and hence flat dispersions, but also in relatively weak bonds that make true stoichiometric compounds difficult to form. Unfortunately $\mathrm{SiC}$, whether $3 \mathrm{C}, 4 \mathrm{H}$ or $6 \mathrm{H}$, has too narrow a phononic gap. Nonetheless $\mathrm{GeC}$ and $\mathrm{SnSi}$ are of significant interest and the former has been grown as a compound, although stoichiometric quality is as yet not high. ${ }^{33,34}$

The IV-VI PbS also has a large mass ratio and is interesting, but its measured phonon dispersion shows an additional TO branch that fills the phonon gap, ${ }^{24}$ thus likely making it unsuitable for an absorber in bulk form. But initial modelling indicates that phonon folding in a nanostructure could reflect these modes and give PbS nano-crystals a large phonon band gap and hence potentially be highly suitable as an absorber. 
There are several other inherent advantages of group IV compounds/alloys all of which are associated with the four valence electrons of the group IVs that result in predominantly covalent bonding:

a) The elements form completely covalently bonded crystals primarily in a diamond structure (tetragonal is also possible as in $\beta \mathrm{Sn}$ ). However for group IV compounds the decreasing electronegativity down the group results in partially ionic bonding. This is not strong in $\mathrm{SiC}$ and whilst it tends to give co-ordination numbers of 4, can nonetheless result in several allotropes of decreasing symmetry: 4C, $4 \mathrm{H}, 6 \mathrm{H}$. However, as the difference in period increases for the group IV compounds, so too does the difference in electronegativity and hence also the bond ionicity and hence the degree of ordering. For a hot carrier absorber this trend is in the right direction because it is just such a large difference in the period that is needed to give the large mass difference and hence large phononic gaps. All of $\mathrm{GeC}, \mathrm{SnSi}, \mathrm{SnC}$ (and the $\mathrm{Pb}$ compounds) have computed phononic gaps large enough to block Klemens decay, and should also tend to form ordered diamond structure compounds.

b) Because of their covalent bonding, the group IV elements have relatively small electronic band gaps as compared to their more ionic III-V and much more ionic II-VI analogues in the same period: e.g. Sn $0.15 \mathrm{eV}$, InSb $0.4 \mathrm{eV}$, CdTe $1.5 \mathrm{eV}$. In fact to achieve approximately the same electronic band gap one must go down one period from group IV to III-V and down another period from III-V to II-VI: e.g. Si $1.1 \mathrm{eV}, \mathrm{GaAs} 1.45 \mathrm{eV}$, CdTe $1.5 \mathrm{eV}$. This means that for group IV compounds there is greater scope for large mass difference compounds whilst still maintaining small electronic band gaps. A small band gap of course being important for broadband absorption in an absorber - property 3 in the desirable properties for hot carrier absorbers listed above.

c) The smaller $\mathrm{E}_{\mathrm{g}}$ would tend to be for the larger mass compounds of $\mathrm{Pb}$ or $\mathrm{Sn}$. Which, to give large mass difference, would be compounded with Si or Ge. This trend towards the lower periods of group IV also means that the maximum optical phonon and maximum acoustic phonon energies will be smaller for a given mass ratio - the desirable properties 4 and 5 .

d) Furthermore, unlike most groups, the group IV elements remain abundant for the higher mass number elements desirable property 9 - in fact it is Ge in the middle of the period that has the lowest moderate abundance. ${ }^{22}$ Property 10 is also satisfied because the group IVs have low toxicity.

\subsubsection{Nanostructures:}

The phonon dispersion of quantum dot (QD) nanostructures can be calculated in a similar way to compounds. ${ }^{6}$ Their phononic properties can be estimated from consideration of their combination force constants. Hence it is possible to 'engineer' phononic properties in a wider range of nanostructure combinations. Of the materials discussed above the Group IVs lend themselves most readily to formation of nanostructures instead of compounds due to their predominantly covalent bonding, which allows variation in the coordination number. Therefore the nanostructure approaches of ${ }^{6}$ are consistent with a similar description as analogues of InN, whether it be III-V QDs, colloidally dispersed QDs or for core shell QDs.

\section{Time Resolved Photoluminescence of Slowed Carrier Cooling}

The effectiveness of InN as a hot carrier absorber material has still to be properly assessed because it is a very difficult material to grow at reasonable quality, again due to the disparity in size of its constituent element atoms. ${ }^{35}$ In addition $\mathrm{InN}$ is predominantly grown as hexagonal wurtzite material rather than the more symmetric cubic zinc-blende structure, although growth of cubic InN is possible.$^{36}$ Some preliminary data on time resolved photoluminescence of InN are shown in Fig. 4 and discussed below.

InP also has a large 'phononic band gap', approximately the same as its maximum acoustic energy, as shown in Fig. 4, re-drawn from. ${ }^{24}$ Also InP predominantly forms as cubic zinc-blende material which is preferable as a hot carrier absorber because of the consequent narrow range of its optical phonon dispersion. The electronic band gap of InP at $1.34 \mathrm{eV}(925 \mathrm{~nm})$ is significantly larger than the optimal hot carrier absorber band-gap but will nonetheless allow absorption of a reasonable part of the solar spectrum. The similarity with the band-gap of GaAs at $1.45 \mathrm{eV}(885 \mathrm{eV})$ makes the comparison of time resolved photoluminescence measurements of this pair of semiconductors useful in order to compare carrier cooling rates of hot carriers. Similar electronic band gaps mean that emission from fully thermalised electron-hole pairs will be at about the same wavelength. This allows direct comparison of hot carrier relaxation plots since for the same excitation wavelength carrier populations with similar temperatures are excited in both semiconductors. Furthermore issues related to differences of calibration of the detectors and non-linear crystal or of differences in chromatic losses of the optical setup are avoided. 


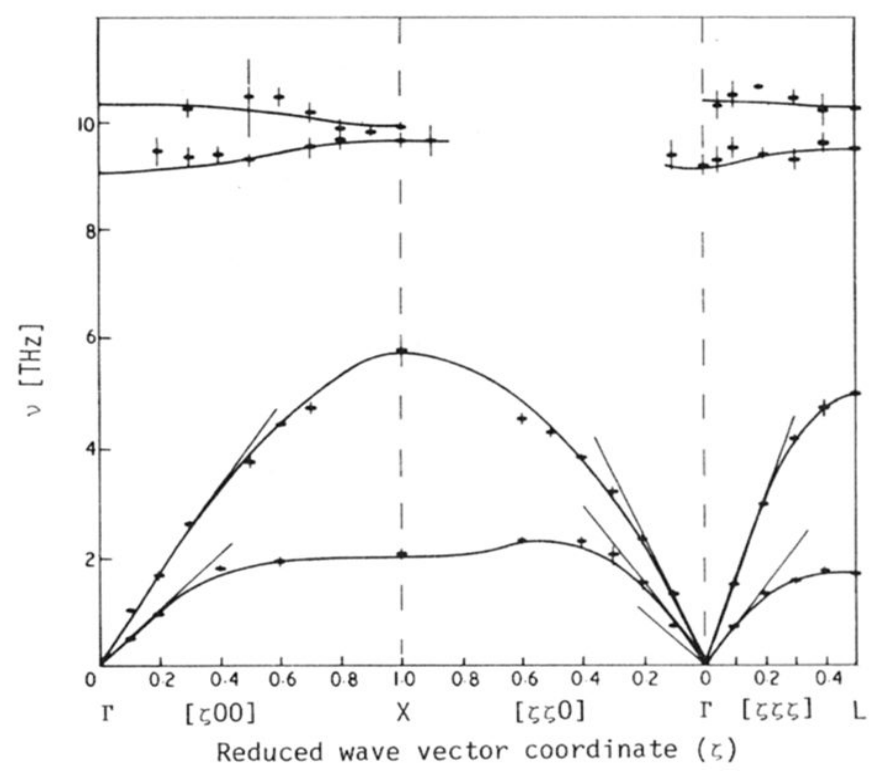

Figure 4: Phonon dispersion for InP showing the large phonic band gap between optical and acoustic modes, redrawn from. ${ }^{24}$

Time resolved photoluminescence (TRPL) measurements comparing GaAs and InP have been carried out in earlier work. ${ }^{11}$ These data are repeated here in Fig. 5 to illustrate the effects of large phonon band gap on carrier cooling. Fig. 5 shows the TRPL spectra emitted by GaAs and InP, with the samples excited by a femtosecond laser pulse at $1.7 \mathrm{eV}(730$ $\mathrm{nm})$. The PL signal is measured from the edge of the band gap to the excitation wavelength with a resolution of $1 \mathrm{ps.}$ The excited carrier density is quite high, $8.510^{19} \mathrm{~cm}^{-3}$ for both semiconductors.

After a fast redistribution over a broad range of energies, hot carriers start to relax towards the band-gap. Fig. 5 clearly shows that carriers in InP thermalise with a longer time constant compared to GaAs. The slower carrier cooling can be attributed to the suppression of the Klemens mechanism in InP due to the larger phononic band-gap that will thus lead to a phonon bottleneck effect and slowed carrier cooling.

The photoluminescence spectra in Fig. 5 were used to fit hot carrier temperatures using a Boltzmann distribution. Fig. 6 shows the evolution of the hot carrier temperature as a function of time for both $\mathrm{InP}$ and GaAs. Calculated relaxation constants are of the order of $500 \mathrm{ps}$ for InP as compared to $200 \mathrm{ps}$ for GaAs. This longer hot carrier lifetime is consistent with the longer phonon lifetimes reported in. ${ }^{11}$ The excitation at $730 \mathrm{~nm}$ is below the conduction band side valleys for both materials, so there is no inter-valley scattering contribution to relaxation, but only $\Gamma$ valley thermalisation, this makes the direct interpretation of slowed carrier cooling in InP valid. Relaxation behaviour for different excitation energies has been investigated and exhibits more complex behaviour, as is reported in. ${ }^{11}$ Hence the evidence presented here supports the supposition that the Klemens mechanism is suppressed in InP.

Also shown in Fig. 6 are temperature TRPL data for a sample of InN. These are very preliminary data as the measurement is not yet properly calibrated, but the data do seem to indicate very high carrier temperatures of $1,000^{\circ} \mathrm{C}$ for short timescales up to 40 ps that are consistent with the high carrier temperatures for InN found in ${ }^{10}$ as shown in Fig. 2. This helps to support the theory of reduced Klemens decay and hence slowed carrier cooling in InN as well as in InP. 

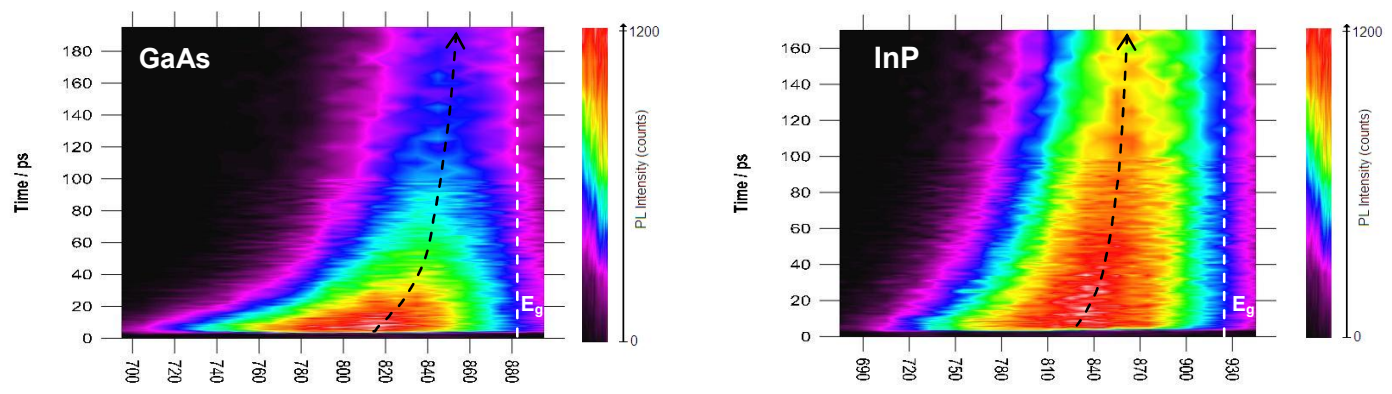

Figure 5: Time resolved PL spectra for bulk (a) GaAs and InP for $730 \mathrm{~nm}$ excitation wavelength redrawn from Clady et al. ${ }^{11}$ Carrier densities are $8.5 \times 1019 \mathrm{~cm}-3$. The peak intensity (black arrows) can be seen to shift closer to the band gap (white lines) with time for both materials, but that for InP stays further above the band gap and at higher intensity for much longer times than that for GaAs.

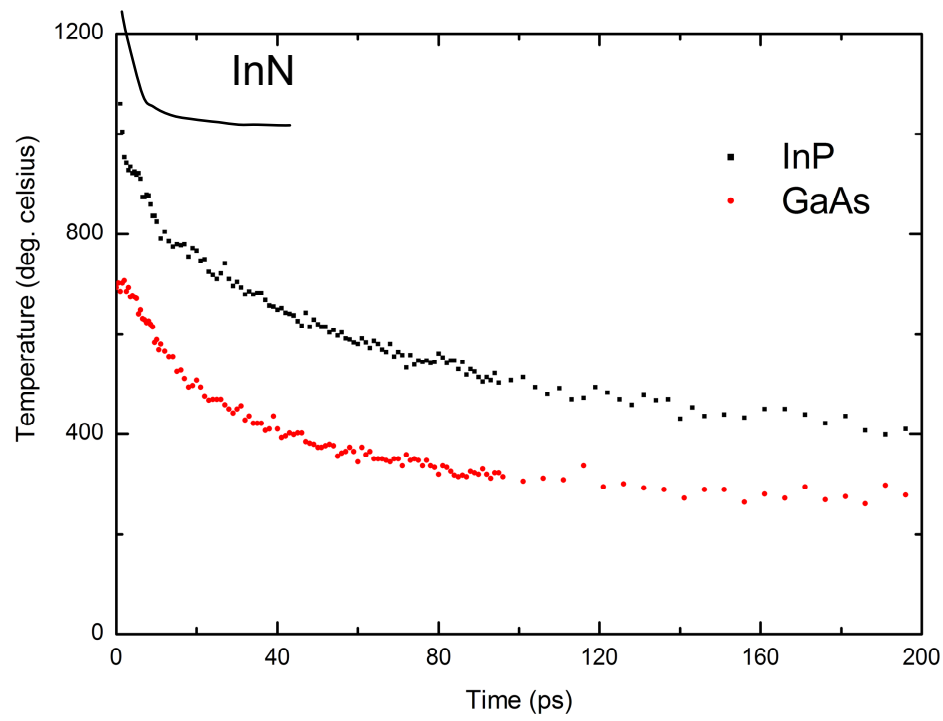

Figure 6: Hot carrier temperature relaxation for bulk GaAs and InP, redrawn from Clady et al. ${ }^{11}$ [Excitation and carrier density are the same as in Fig. 5.] Also shown in the top left are calculated (although incompletely calibrated) carrier temperatures from TRPL data for InN, which indicate high carrier temperatures at short times.

\section{Discussion and Conclusion}

The Hot Carrier solar cell has the potential to achieve very high efficiencies in a device that is essentially a single junction. Detailed balance calculations indicate limiting efficiencies as high as $65 \%$ under 1 sun and $85 \%$ under maximum concentration. However a series of modelling developments has shown that as real material parameters are introduced the predicted efficiencies decrease. What emerges is that maximisation of the thermalisation time constant for hot carriers is critical to improved efficiency.

The carrier cooling mechanisms depend primarily on emission of optical phonons by cooling carriers, predominantly electrons. Under some circumstances these optical phonons can be produced at such a high density that they cannot decay away fast enough and a 'phonon bottleneck' is formed that allows the phonon energy to scatter back with the electron ensemble thus re-heating it. Creating the conditions for this phonon bottleneck seems the most fruitful route for significantly increasing the thermlaisation time constant.

Quantum well nanostructures exhibit such phonon confinement with significantly hot carrier temperatures. The reasons for this are not completely clear but are affected by the restriction of hot carriers diffusing in the direction perpendicular to the wells and by confinement of phonons in the wells. Prevention of decay of optical phonons into acoustic phonons is another method for maximising phonon bottleneck. Materials with a large difference in acoustic and optical phonon 
energies can block this Klemens route for phonon decay. A number of materials are identified as having these properties with the principle requirement that there is a large mass difference between their constituent atoms. Some of the most promising are III-nitrides, especially InN, and their analogues, which include transition metal nitrides - of which HfN and $\mathrm{ZrN}$ are most interesting - and group IV compounds - of which $\mathrm{SnSi}$ has the most impressive modelled properties. Experimental demonstration of these effects are limited at present although time resolved photoluminescence measurements of carrier temperature suggest that long hot carrier lifetimes are achieved in both InP and InN, both of which have large phonon band gaps. There are encouraging signs that these properties will soon be demonstrated in several material groups. Combinations of materials with large phonon band gaps in nano-well nanostructures offer great potential to combine two slowing of carrier cooling mechanisms and hence achieve higher longer lived hot carrier temperatures.

\section{Acknowledgements}

This work has been supported by the Australian Government through the Australian Renewable Energy Agency (ARENA). Responsibility for the views, information or advice expressed herein is not accepted by the Australian Government.

GC acknowledges the support of the Australian Research Council through its Future Fellows scheme.

\section{References}

[1] Green, M. A.' [Third Generation Photovoltaics: Ultra-High Efficiency at Low Cost], Springer-Verlag, (2003).

[2] Würfel, P., Sol. Energy Mats. and Sol. Cells, 46, 43 (1997).

[3] Ross, R., Nozik, v, J. Appl. Phys, 53, 3318 (1982).

[4] Shrestha, S.K., Aliberti, P., Conibeer, G.J., “Energy selective contacts for hot carrier solar cells,” Solar Energy Materials and Solar Cells, 94, 1546-1550 (2010).

[5] Le Bris, A., Guillemoles, J-F., Appl. Phys. Lett., 97, 113506 (2010); LeBris, A., [PhD Thesis] UPMC, Paris, (2010).

[6] Patterson, R., Kirkengen, M., Puthen Veettil, B., Konig, D., Green, M.A., Conibeer, G., "Phonon lifetimes in model quantum dot superlattice systems with applications to the hot carrier solar cell," Solar Energy Materials and Solar Cells, 94, 1931-1935 (2010).

[7] Feng, Y., Patterson, R., Lin, S., Shrestha, S., Huang, S., Green, M.A., Conibeer, G, "Investigation of carrier-carrier scattering effect on the performance of hot carrier solar cells with relaxation time approximation", Appl. Phys. Lett., accepted May 2013. DOI: 10.1063/1.4811263 (2013).

[8] Klemens, P.G., Phys. Rev., 148, 845 (1966).

[9] Conibeer, G., Patterson, R., Aliberti, P., Huang, L., Guillemoles, J-F., König, D., Shrestha, S., Clady, R., Tayebjee, M., Schmidt, T., Green, M.A., Proc. 24th European Photovoltaic Solar Energy Conference, Hamburg, Hot Carrier solar cell Absorbers (2009).

[10] Chen, F., Cartwright, A.N., Appl Phys Lett, 83, 4984 (2003).

[11] Clady, R., Tayebjee, M.J.Y., Aliberti, P., Konig, D., Ekins-Daukes, N.J., Conibeer, G.J., Schmidt, T.W., Green, M.A., "Interplay between Hot phonon effect and Intervalley scattering on the cooling rate of hot carriers in GaAs and InP”, Prog. Photovolt: Res. Appl., 20, 82-92 (2012).

[12] Rosenwaks, Y., Hanna, M., Levi, D., Szmyd, D., Ahrenkiel, R., Nozik, A., Phys. Rev. B, 48, 14675-14678 (1993).

[13] Guillemoles, J-F., Conibeer, G., Green, M.A., Proc. 21st European Photovoltaic Solar Energy Conference, Dresden, 234-237 (2006).

[14] Hirst, L., Führer, M., LeBris, A., Guillemoles, J-F., Tayebjee, M., Clady, R., Schmidt, T., Wang, Y., Sugiyama, M., Ekins-Daukes, N., Proc. 37th IEEE Photovoltaics Specialists Conference, Seattle, talk 927 (2011). 
[15] Hirst, L.C., Fuhrer, M., Farrell, D.J., Le Bris, A., Guillemoles, J-F., Tayebjee, M.J.Y., Clady, R., Schmidt, T.W., Sugiyama, M., Wang, Y., Fujii, H., Ekins-Daukes, N.J., Proc. SPIE Photonics West conf., San Francisco,

"InGaAs/GaAsP quantum wells for hot carrier solar cells" (2012).

[16] Hirst, L.C., Walters, R. J. F€ uhrer, M. F., Ekins-Daukes, N. J., "Experimental demonstration of hot-carrier photocurrent in an InGaAs quantum well solar cell”, Appl. Phys. Lett., 104, 231115 (2014).

[17] Colvard, C., Gant, T.A., Klein, M.V., Phys. Rev. B, 31, 2080-2091 (1985).

[18] Todorov, T.K., Reuter, K.B., Mitzi, D.B., Advanced Materials, 22, 1-4 (2010).

[19] Lambrecht, W.R. L., Phys. Rev. B, 72, 155202 (2005).

[20] Erwin, S.C., Zutic, I., Nature Materials, 3, 410-414 (2004).

[21] Paudel, T.R., Lambrecht, W.R.L., Phys. Rev. B, 79, 245205 (2009).

[22] Cox in [The Elements: Their Origin, Abundance, and Distribution], Oxford University Press, (1989); [McGraw-Hill Encyclopedia of Science \& Technology], 7th ed., McGraw-Hill, New York USA, (1992): James, A.M., Lord, M.P., in [Macmillan's Chemical and Physical Data], Macmillan, London UK, (1992).

[23] König, D., Casalenuovo, K., Takeda, Y., Conibeer, G., Guillemoles, J-F., Patterson, R., Huang, L., Green, M.A., "Hot Carrier Solar Cells: Principles, Design and Materials", Physica E, 42, 2862-2866 (2010).

[24] Bilz, H., Kress, W., [Phonon dispersion relations in Insulators], Springer, (1979).

[25] Tea, E., Hamzeh, H., Aniel, F., J. Appl. Phys., 110 , 113108 (2011); Tea, E., Levard, H., Hamzeh, H., Le Bris, A., Laribi, S., Aniel, F., Guillemoles, J-F., "Advanced modeling of Hot carrier effects in 3rd generation solar cells", Proc. 27th Euro PVSEC, Frankfurt, p 516 (2012); Guillemoles, J-F., Le Bris, A., Lombez, L., Laribi, S., Tea, E., Aniel, F., "Hot carrier solar cells: The device that did not exist (but should)", in: [Numerical Simulation of Optoelectronic Devices (NUSOD)], 11th International Conference, p17 (2011).

[26] Barman, S., Shrivestava, G.P., Phys. Rev., 69, 235208 (2004).

[27] Yao, Y., Konig, D., Green, M.A., Solar Energy Materials and Solar Cells, 111, 123-126 (2013).

[28] Dalui, S., Das, S.N., Hussain, S., Paramanik, D., Verma, S., Pal, A.K., J. Crystal Growth, 305(1), 149-155 (2007); Hussain, S., Dalui, S. Roy, R.K., Pal, A.K., J. Physics D: Applied Physics, 39, 2053 (2006).

[29] Le Bris, A., Lombez, L., Laribi, S., Boissier, G., Christol, P., Guillemoles, J-F., "Thermalisation rate study of GaSbbased heterostructures by continuous wave photoluminescence and their potential as hot carrier solar cell absorbers", Energy Environ. Sci., 5, 6225 (2012).

[30] Saha, B., Sands, T.D., Waghmare, U.V., J. Appl. Phys., 109, 073720 (2011).

[31] Saha, B., Acharya, J., Sands, T.D., Waghmare, U.V., J Appl. Phys., 107, 033715 (2010).

[32] Norlund-Christensen, A., Kress, W., Miura, M., Phys. Rev. B, 28, 977 (1983).

[33] Liu, Z.T., Zhu, J.Z., Xu, N.K., Zheng, X.L., Jap. J. Appl. Phys., 36, 3625 (1997).

[34] Gupta, N., Shrestha, S., Conibeer, G., Xia, H., Puthen-Veettil, B., Proc. 28th European Photovoltaic Solar Energy Conference, Paris, "Crystallographic analysis of GexC1-X film deposited by RF Magnetron Sputtering for Hot Carrier Solar Cells", (2013).

[35] Conibeer, G.J., König, D., Green, M.A., Guillemoles, J-F., "Slowing of carrier cooling in Hot Carrier solar cells", Thin Solid Films, 516, 6948 (2008).

[36] Cimalla, V., et al., "Growth of cubic InN on r-plane sapphire”, Applied Physics Letters, 83(17), 3468-3470 (2003). 